\title{
Gastrointestinal stromal tumour treated with neoadjuvant imatinib
}

\author{
M B Loughrey, C Mitchell, G B Mann, M Michael, P M Waring
}

J Clin Pathol 2005;58:779-781. doi: 10.1136/icp.2004.023226

This report describes a case of unresectable primary gastrointestinal stromal tumour (GIST) treated with imatinib on a neoadjuvant basis, before subsequent successful surgical resection. After six months of imatinib, computed tomography and positron emission tomography imaging demonstrated a significant size reduction and complete metabolic response to treatment, rendering the tumour resectable. Mutational analysis showed an activating KIT mutation in exon 11. The pathological appearance of the resected tumour was heterogeneous with extensive necrosis, cystic and myxoid change, extensive hypocellularity, and patchy foci of residual viable tumour. The implications for this management option of radiological, pathological, and molecular assessment are discussed.

G astrointestinal stromal tumours (GISTs) are rare, mesenchymal tumours of the gastrointestinal tract driven by activated KIT oncoprotein, a type III receptor tyrosine kinase. $^{1}$ Mutations in the KIT gene are the most common cause of activation. Imatinib mesylate (Glivec ${ }^{\circledR}$ / Gleevec ${ }^{\circledR}$, formerly STI571; Novartis Pharma AG, Basel, Switzerland) is a selective tyrosine kinase inhibitor that inhibits the mutated KIT oncoprotein. Imatinib has been used to treat patients with metastatic or unresectable GIST in phase I and II trials with considerable success. Phase II and III adjuvant imatinib trials and a single phase II neoadjuvant imatinib trial are under way. ${ }^{2}$ We report a case of unresectable primary GIST treated with imatinib on a neoadjuvant basis, before subsequent surgical resection.

\begin{abstract}
"Gastrointestinal stromal tumours are rare, mesenchymal tumours of the gastrointestinal tract driven by activated KIT oncoprotein, a type III receptor tyrosine kinase"
\end{abstract}

\section{CASE REPORT}

A 69 year old man presented with worsening abdominal swelling, dysphagia, and weight loss. Computed tomography (CT) and positron emission tomography (PET) scans revealed a $170 \mathrm{~mm}$ diameter metabolically active mass in the left upper abdominal quadrant (fig 1A, C). No metastases were evident. Needle core biopsy showed a tumour composed of interlacing fascicles of spindle cells with elongated nuclei and weakly eosinophilic cytoplasm. Immunohistochemistry revealed membranous CD117 (KIT) positivity, confirming a diagnosis of GIST. Automated sequencing of KIT exon 11 identified a GAA $\rightarrow$ TCC trinucleotide substitution at base position 1671-1673, resulting in a WK $\rightarrow$ CP double amino acid change at residues 557 and 558.

The tumour was considered unresectable and treatment was begun with imatinib $400 \mathrm{mg} /$ day. A rapid clinical response followed. Restaging scans were performed after three and six months. CT revealed a reduction in tumour diameter of approximately $50 \%$ after six months (fig 1B). Both PET scans showed a total loss of $\left[{ }^{18} \mathrm{~F}\right]$ fluorodeoxyglucose uptake, indicating a sustained complete metabolic response (fig lD). At this stage, the tumour was considered resectable. At laparotomy, the tumour was soft but well encapsulated, and arose from a narrow base in the anterior wall of the stomach. Complete surgical resection without tumour rupture was performed successfully with a partial gastrectomy.

Pathological examination of the resection specimen revealed a lobulated, pale grey tumour measuring $120 \mathrm{~mm}$ in maximum diameter (fig 2A). The tumour was heterogeneous with extensive gelatinous change, cystic degeneration, a large central necrotic area, and nodules of more solid, fleshy tissue. Microscopy confirmed widespread myxoid degeneration with cystic change, necrosis, and hypocellularity (fig 2B, C). Nodules of residual viable CDll7 positive tumour cells made up approximately $10 \%$ of the overall tumour volume (fig 2D). Mutational analysis repeated using DNA extracted from residual tumour tissue showed the same KIT exon 11 mutation, but no acquired KIT mutations, in exons 11 and 17.

The patient made a rapid recovery postoperatively. Follow up CT and PET scans at four weeks confirmed no residual disease and imatinib treatment was stopped. The patient will continue with surveillance imaging at three monthly intervals initially, with reintroduction of imatinib should disease recur.

\section{DISCUSSION}

Reports of GIST resection after treatment with imatinib are limited. One series describes 17 patients with initially unresectable GISTs who underwent tumour resection after a median of 10 months of treatment with imatinib. ${ }^{3}$ Thirteen patients had a partial (11) or complete (two) pathological response, based on evaluation of residual viable tumour cells. Hohenberger et al recently reported 18 patients with GIST treated surgically after imatinib pretreatment. ${ }^{4}$ Patients with evidence of a partial response at the time of surgery had a much better outcome than those with progressive disease. Two other reports describe the successful use of neoadjuvant imatinib in one and two cases, with complete resection of tumour after partial response to the drug. ${ }^{56}$ One case was reported to have a KIT exon 11 W557R missense mutation. ${ }^{6}$ Pathological tumour changes described after treatment with imatinib were similar to those seen in our case, namely tumour necrosis, hypocellularity, myxoid change, and sclerosis. $^{7}$

Recent series looking at the prognostic value of KIT mutations in GISTs treated with imatinib alone have reported

Abbreviations: $\mathrm{CT}$, computed tomography; GIST, gastrointestinal stromal tumour; PET, positron emission tomography 


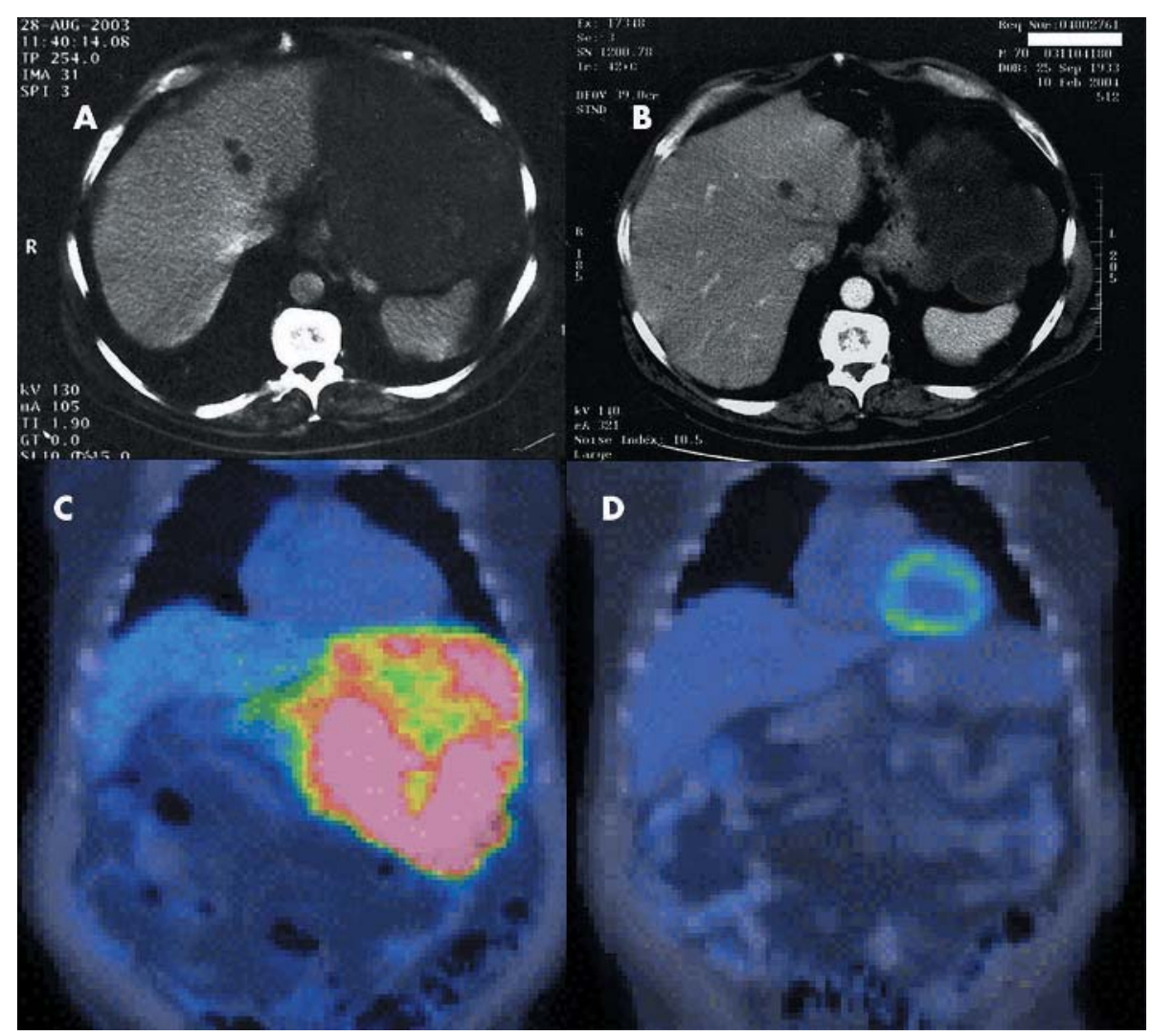

Figure 1 Computed tomography (CT) and positron emission tomography (PET) imaging response to imatinib in a patient with advanced, gastric gastrointestinal stromal tumour: (A, C) before imatinib treatment, (B, D) after six months of imatinib $400 \mathrm{mg} /$ day. Note the approximately $50 \%$ reduction in size on $\mathrm{CT}$ imaging (B) and total loss of $\left[{ }^{18} \mathrm{~F}\right]$ fluorodeoxyglucose uptake on PET scan (D), indicating a complete metabolic response.

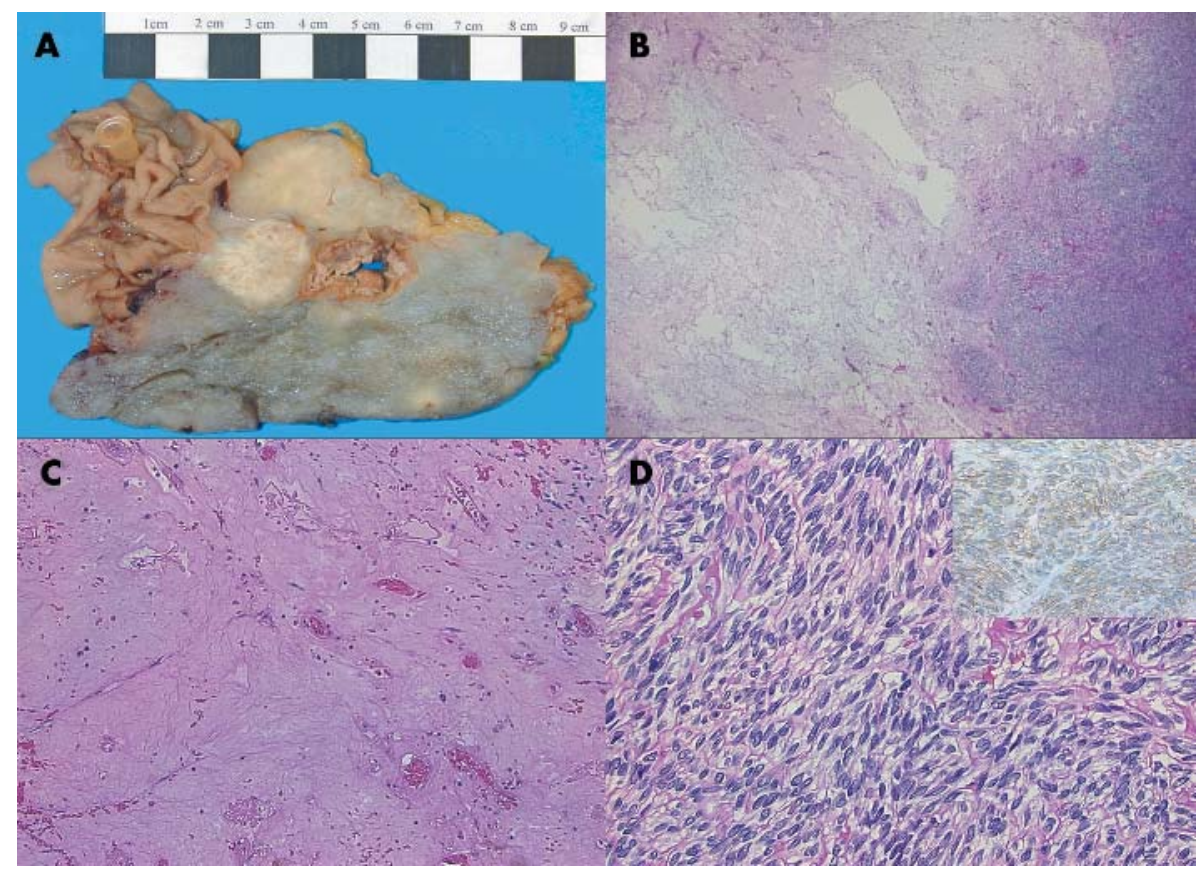

Figure 2 (A) Gastrointestinal stromal tumour (GIST) resection specimen after treatment with imatinib, macroscopically showing normal gastric mucosa (top left) and underlying heterogeneous tumour with extensive gelatinous change, a central necrotic area, and nodules of solid pale tissue containing residual viable tumour. (B-D) Representative GIST tumour sections. (B) Low power view showing cystic change and adjacent residual tumour (right). (C) Myxoid degeneration and hypocellularity. (D) Residual viable spindle shaped tumour cells, identical to pretreatment biopsy (haematoxylin and eosin stain; original magnification, $\times 100-200$ ). (Inset) Diffuse membranous positivity for CD117 (KIT) in tumour cells (CD117 immunohistochemistry; original magnification, $\times 200$ ). 


\section{Take home messages}

- This case report supports the use of neoadjuvant imatinib in unresectable or marginally resectable primary gastrointestinal stromal tumours

- Surgical intervention should be timed towards the period of maximal tumour response but before disease progression occurs as a result of acquired secondary KIT mutations

- Although treatment induced size reduction may improve the likelihood of successful tumour resection, tumour necrosis and myxoid change may increase the risk of tumour rupture

- Preoperative KIT mutation analysis may predict the likelihood of tumour response to imatinib treatment

an increased likelihood of partial response and longer median survival times in GISTs harbouring exon 11 mutations compared with all others (non-exon 11 mutations and wild type) ${ }^{8-10}$ Most KIT exon 11 mutations in GISTs are in frame deletions involving the region between residues 553 and 561, but missense mutations, including those of several adjacent bases causing double amino acid substitutions, have been reported, mainly at the 557-558 hotspot. ${ }^{11}$ In the neoadjuvant setting, preoperative genotyping may give an indication of the likely response to imatinib and could be used in developing a treatment plan. However, it has been shown that, after a good initial response to treatment, exon 11 mutated GISTs treated with imatinib may develop disease progression, usually because of the acquisition of secondary KIT mutations, conferring drug resistance. ${ }^{12}$ Hence, the timing of surgical intervention after neoadjuvant imatinib is important. In most GISTs that respond to imatinib, the initial response lasts at least 12 months before progression occurs. Surgical intervention should be targeted towards this window, aiming at the time of maximal response but before progression occurs. Close monitoring with CT and PET scanning during this period is important. No secondary KIT mutation was found in our case in the residual tumour tissue unresponsive to imatinib treatment.

"In the neoadjuvant setting, preoperative genotyping may give an indication of the likely response to imatinib and could be used in developing a treatment plan"

Often, the assessment of resectability on the basis of CT scanning with or without laparoscopy is imperfect. In cases of doubtful or marginal resectability, preoperative imatinib may render a tumour clearly resectable. Tumour rupture is known to be associated with a high risk of disseminated intraperitoneal recurrence. A major reduction in tumour size may make rupture less likely, although the treatment induced necrosis and myxoid changes may increase this risk.
Our case of partial response to imatinib treatment and successful resection of a previously unresectable primary GIST supports the use of imatinib in the neoadjuvant setting. Consideration should be given to preoperative KIT mutation analysis if available. The outcome of prospective randomised trials evaluating this management strategy is awaited.

\section{ACKNOWLEDGEMENTS}

M B Loughrey is supported by a Cancer Council Victoria research scholarship.

\section{Authors' affiliations}

M B Loughrey, C Mitchell, P M Waring, Department of Pathology, Peter MacCallum Cancer Centre, Melbourne, VIC 3002, Australia

G B Mann, Department of Surgical Oncology, Peter MacCallum Cancer Centre

M Michael, Department of Medical Oncology, Peter MacCallum Cancer Centre

Correspondence to: $\operatorname{Dr}$ M B Loughrey, Department of Pathology, Peter MacCallum Cancer Centre, St Andrew's Place, East Melbourne, VIC 3002, Australia; mauriceloughrey@doctors.org.uk

Accepted for publication 1 November 2004

\section{REFERENCES}

1 Fletcher CD, Berman JJ, Corless C, et al. Diagnosis of gastrointestinal stromal tumors: a consensus approach. Hum Pathol 2002;33:459-65.

2 Eisenberg BL, Judson I. Surgery and imatinib in the management of GIST: emerging approaches to adjuvant and neoadjuvant therapy. Ann Surg Oncol 2004; 11:465-75.

3 Scaife $\mathrm{CL}$, Hunt $\mathrm{KK}$, Patel SR, et al. Is there a role for surgery in patients with "unresectable" cKIT+ gastrointestinal stromal tumors treated with imatinib mesylate? Am J Surg 2003;186:665-9.

4 Hohenberger $P$, Bauer S, Schneider $U$, et al. Tumor resection following imatinib pretreatment in Gl stromal tumors [abstract 3288]. Proceedings of the American Society of Clinical Oncology 2003;22:818.

5 Katz D, Segal A, Alberton Y, et al. Neoadjuvant imatinib for unresectable gastrointestinal stromal tumor. Anticancer Drugs 2004;15:599-602.

6 Bumming P, Andersson J, Meis-Kindblom JM, et al. Neoadjuvant, adjuvant and palliative treatment of gastrointestinal stromal tumours (GIST) with imatinib: a centre-based study of 17 patients. $\mathrm{Br} J$ Cancer 2003;89:460-4.

7 Joensuu H, Roberts PJ, Sarlomo-Rikala M, et al. Effect of the tyrosine kinase inhibitor STI571 in a patient with a metastatic gastrointestinal stromal tumor. N Engl J Med 2001;344:1052-6.

8 Heinrich MC, Corless CL, von Mehren M. PDGFRA and KIT mutations correlate with the clinical responses to imatinib mesylate in patients with advanced gastrointestinal stromal tumors (GIST) [abstract 3274]. Proceedings of the American Society of Clinical Oncology 2003;22:815.

9 Heinrich MC, Corless CL, Demetri GD, et al. Kinase mutations and imatinib response in patients with metastatic gastrointestinal stromal tumor. J Clin Oncol 2003;21:4342-9.

10 Debiec-Rychter M, Dumez H, Judson I, et al. Use of c-KIT/PDGFRA mutational analysis to predict the clinical response to imatinib in patients with advanced gastrointestinal stromal tumours entered on phase I and II studies of the EORTC soft tissue and bone sarcoma group. Eur J Cancer 2004;40:689-95.

11 Antonescu CR, Sommer G, Sarran L, et al. Association of KIT exon 9 mutations with nongastric primary site and aggressive behavior: KIT mutation analysis and clinical correlates of 120 gastrointestinal stromal tumors. Clin Cancer Res 2003:9:3329-37.

12 Fletcher JA, Corless CL, Dimitrijevic S. Mechanisms of resistance to imatinib mesylate (IM) in advanced gastrointestinal stromal tumor (GIST) [abstract 3275]. Proceedings of the American Society of Clinical Oncology 2003;22:815. 